$\mathrm{dG}$ opposite $\mathrm{\kappa}$ was observed, and $\chi$ was not incorporated in positions opposite to bases other than $\mathrm{K}$ if the full set of deoxynucleoside triphosphates was available. Transcription using T7 RNA polymerase is more error-prone, but this is not surprising because RNA polymerases are usually less accurate than DNA polymerases. The demonstration that the incorporation of $\chi$ in the template leads to the faithful incorporation of $\kappa$ in the product would complete the proof that an extended genetic alphabet is possible.

The pyrimidine analogues discussed, with the exception of iso-C, are substances in which the base and sugar are joined by a carbon-carbon bond. Iso-C and iso-G do not form a completely satisfactory base pair (see above), which perhaps accounts for their 'rejection' by evolution. Perhaps the other base pairs were not used because methods for the prebiotic synthesis or early biosynthesis of the pyrimidine analogues were not available.

The incorporation of $\chi$ as one of the main constituents of a replicating nucleic acid might present special problems because, at near neutral pHs, the base carries a negative charge that is not present on the standard nucleosides. It would be interesting to study the stability of the double helices formed by oligomers containing a substantial proportion of xanthosine. Incidentally, it is perhaps surprising that DNA and RNA polymerases accept $\chi \mathrm{TP}$ as normal substrate, despite the extra charge that it carries.

Piccirilli et al. expect that the expansion of the genetic alphabet from four to six or more letters will enable them to incorporate functionalized monomers into oligonucleotides with extended catalytic capacity. This is an interesting suggestion, but it is not obvious to me that a similar result could not be achieved using chemically synthesized oligomers based on functionalized derivatives of the standard nucleotides. The authors also suggest that, in the long run, the genetic code can be extended artificially. How many amino acids can be coded for with triplets derived from a 12-letter alphabet? Don't forget to take account of wobble!

Leslie E. Orgel is at The Salk Institute for Biological Studies, PO Box 85800, San Diego, California 92138, USA

1. Piccirilli. J.A., Krauch, T., Moroney, S.E. \& Benner, S.A. Nature 343, 33-37 (1990).

2. Rich, A. in Horizons in Biochemistry (eds Kasha, M. \& Pullman, B.) 103 (Academic, New York, 1962).

3. Switzer, C.Y., Moroney, S.E. \& Benner, S.A. J. Am. chem Soc. 111, 8322 (1989)

\title{
A case of male opportunism
}

\section{Malte Andersson}

THE idea that male secondary sex traits evolve through female choice has been controversial since Darwin' first proposed it. Over a century later it remains so, as discussed in a News and Views article four years ago $^{2}$. The two most debated explanations $^{3-5}$ are Fisher's ${ }^{6}$ runaway process and various forms of indicator mechanism, both of which assume that male trait and female preference evolve together. On page 66 of this issue ${ }^{7}$, Ryan et al. report that neither applies to the case of the tungara frog (Physalaemus pustulosus). Females are attracted by a male call, but their preference has not evolved together with it; rather, the call seems to take advantage of a pre-existing frequency bias of the female ear. If similar mechanisms apply in many species, present views of sexual selection by female choice may have to be changed.

In some animals, female mate choice can be explained by advantages in the form of resources provided by the male, which enable the female to have more offspring $^{8}$. In others, males contribute nothing but genes. For such animals, the fisherian explanation holds that the female preference evolves together with the preferred male trait in a process that becomes self-reinforcing. It can therefore bring the male trait to an extreme stage of development. Indicator mechanisms, on the other hand, suggest that the female preference evolves together with a male trait that advertises high heritable viability.

Ryan et al.'s conclusion - that in the tungara frog the female preference remains unaffected by the evolution of the male call - was reached by comparing $P$. pustulosus with a closely related frog, $P$. coloradorum. The two species show similar frequency tuning of the basilar papilla, the main sound receptor stimulated by the 'chuck' part of the male call. Yet $P$. coloradorum does not produce chucks. The simplest explanation seems to be that the similar basilar papilla tuning in the two species has been inherited from a common ancestor. If so, the female preference for chucks in $P$. pustulosus has not evolved together with the male call. The call instead appears to be an opportunistic adaptation that takes advantage of the given bias of the female ear.

The results help explain why females in this species favour low-frequency calls, and why large males, which produce such calls, have a mating advantage. Audiograms show that the sensitivity of the female basilar papilla is maximal for calls of lower frequency than those of the average male. Calculations based on properties of the female auditory system and the frequency spectrum of male calls indicate that lowered fundamental frequency of the chuck call will stimulate females more effectively. For these reasons, sexual selection of the call characteristics and body size in male tungara frogs seems to stem, at least in part, from the properties of the female ear.

At least two questions are raised by these results. First, the fisherian model predicts that the female preference evolves along with the male trait in polygynous species - so why has this not occurred to any appreciable degree in the tungara frog? One possibility is that there has not been sufficient genetic variation in the preference. Second, what factors have selected for the present frequency tuning of the female (and perhaps male) ear? Further analysis of these aspects would be of interest.

Fisher formulated his runaway model of female choice to explain how sexual selection can lead to extreme sex traits that reduce male survival. The chuck call of the male tungara frog means the frogs risk predation by frog-eating bats ${ }^{9}$, and is therefore precisely the kind of sex trait that made Fisher (and Darwin) arrive at their ideas on sexual selection. But the evidence presented by Ryan et al. suggests that the chuck call has not evolved by runaway selection. Also, in some other animals, conspicuous male sex ornaments have been shown to be selected in other ways $^{10}$. The complex process envisaged by Fisher will perhaps turn out to be less important than has been thought, and simpler mechanisms may account for even the most conspicuous secondary sex signals.

That is by no means certain, however. The possibility of genetic coevolution of the preference and the preferred trait has been supported by other studies, for example of ladybirds ${ }^{11}$. Much more work remains to be done before a reliable general picture of the evolution of secondary sex traits by mate choice will emerge.

Malte Andersson is in the Department of Zoology, University of Gothenburg, PO Box 25059, 40031 Gothenburg, Sweden.

1. Darwin, C. The Descent of Man, and Selection in Relation to Sex (Murray, London, 1871)

2. Partridge, L. \& Harvey, P. Nature 323, 580-581 (1986).

3. Bradbury, J.W. \& Andersson, M.B. Sexual Selection: Testing the Alternatives (Wiley, Chichester, 1987).

4. Kirkpatrick, M. A. Rev. Ecol. Syst. 16, 43-70 (1987).

5. Pomiankowski, A.N. Oxford Surv. Evol. Biol. 5, 136-184 (1988).

6. Fisher. R.A. The Genetical Theory of Natural Selection (Clarendon, Oxford, 1930)

7. Ryan, M.J., Fox, J.H., Wilczynski, W. \& Rand, A.S. Nature 343, 66-67 (1990)

8. Alcock, J. Animal Behavior: An Evolutionary Approach, 4th edn (Sinauer, Sunderland, 1989).

9. Tuttle, M.D. \& Ryan, M.J. Science 214, 677-678 (1981).

10. Butcher, G.S. \& Rohwer. S. Curr. Ornithol 6, 51-108 (1988).

11. Majerus, M.E.N., O'Donald, P., Kearns, P.E.W. \& Ireland, H. Nature 321, 164-167 (1986). 